\title{
Experimental Model with Choledochojejunostomy in Rats-Technique and Significance
}

\author{
Naoki Hashimoto ${ }^{1 *}$, Shigeaki Yamanaka ${ }^{2}$, Haruhiko Imamoto ${ }^{1}$ \\ ${ }^{1}$ Department of Surgery, Kindai University, Osaka, Japan \\ ${ }^{2}$ Life Science Institute, Kindai University, Osaka, Japan \\ Email: ^gojigen000@gmail.com
}

How to cite this paper: Hashimoto, N., Yamanaka, S. and Imamoto, H. (2016) Experimental Model with Choledochojejunostomy in Rats-Technique and Significance. Open Access Library Journal, 3: e3217. http://dx.doi.org/10.4236/oalib.1103217

Received: November 9, 2016

Accepted: November 21, 2016

Published: November 24, 2016

Copyright $\odot 2016$ by authors and Open Access Library Inc.

This work is licensed under the Creative Commons Attribution International

License (CC BY 4.0).

http://creativecommons.org/licenses/by/4.0/

\begin{abstract}
A simple technique of choledochojejunostomy in rats is introduced in this paper and its suitability for use is as an experimental model. The healing characteristics of sutureless choledochojejunostomy rat (C-J rat) were investigated in a rat experimental model. The common bile duct of 10 conditioned Wistar rats was exposed, divided transversely and a choledochojejunostomy constructed with only a vinyl chloride tube positioned between the common bile duct and jejunum. The animals were sacrificed on 12 weeks after surgery. From the serum biochemical examination on 12 weeks after surgery, serum bilirubin, AST and ALT in C-J rat were similar to those of control. But Alp in C-J rat is significantly increased compared to control due to stent tube. Therefore, there is no problem about the bile flow via choledochojejunostomy in C-J rat. The results of the present study indicate clearly that the sutureless anastomosis in a rat model is reliable and leads to long-term anastomotic patency at 12 weeks after surgery. Sutureless choledochojejunostomy may have an application in the reconstruction of narrow and fragile bile duct. Our technique will be suitable for the cases of narrow and fragile bile duct which standard biliary enteric anastomosis is impossible.
\end{abstract}

Subject Areas

Gastroenterology \& Hepatology

\section{Keywords}

Choledochojejunostomy, Sutureless

\section{Introduction}

Bilioenteric anastomosis has played an integral role in the surgical management of bi- 
liary tract disease. Although various methods of biliary reconstruction have been reported, they are often associated with troublesome complications, such as anastomotic leakage, stricture, cholangitis, and postoperative cholelithiasis. Especially, bilioenteric anastomosis in rats is technically difficult, due to the small diameter of the bile duct.

The purpose of this work was to evaluate the suitability of rats with a bilioenteric anastomosis produced by a simple method. A simple method of biliary reconstruction has been devised that uses only a stent tube positioned between the common bile duct and jejunum, without suturing them together.

\section{Material and Methods}

\subsection{Animals}

Fifteen male Wistar rats, 8 weeks old, weighing around 200 - 250 g, were used for this experiment. They were purchased from the Experimental Animal Center of CLEA Japan Inc. They were housed in wide-mesh wire cages and fed on a standard pellet diet with water. Food, but not water, was withheld for $24 \mathrm{~h}$ before the surgical procedure and reinstituted $24 \mathrm{~h}$ later. The animals were weighed weekly. Any animal becoming moribund during the course of the experiment was sacrificed by an overdose of Somnobentyl.

\subsection{Surgical Procedure}

Choledocho-jejunostomy rat (C-J rat)

The technique of biliary duct implantation in the small bowel was done as follows.

Laparotomy was carried out under general anesthesia. The bile duct was exposed, divided and ligated distally with two 5-0 vicryl ligatures. A vinyl chloride tube (length 30 $\mathrm{mm}$, external diameter $0.61 \mathrm{~mm}$, internal diameter $0.28 \mathrm{~mm}$, Becton Dickinson and Company) was used as a stent. One end of the tube was inserted into the divided CBD 5 $\mathrm{mm}$ distal to the hepatic confluence and ligated in place with 5-0 vicryl (Figure 1). The other end was inserted into the $18 \mathrm{G}$ needle which was inserted through the jejunum from the mesenteric side to the antimesenteric side of the jejunal lumen $25 \mathrm{~cm}$ distal to the ligament of Treitz (Figure 2). After the $18 \mathrm{G}$ needle was removed from the jejunum, proximal end of the tube held in place with a purse-string suture with 5-0 nylon, which was ligated with 5-0 vicryl in common bile duct (Figure 3). The ligated CBD tube brought out through the mesenteric side of the jejunum was cut just enough to re-establish bile flow. The cut end spontaneously retracted into the intestinal lumen. And the hole in the jejunum was closed with a stich using 5-0 nylon (Figure 4).

\subsection{Experimental Design}

A group of ten rats were performed Choledochojejunostomy (C-J rat). A group of five rats subjected to sham operation served as a control. They were sacrificed on 12 weeks.

\subsection{Serum Biological Examination}

At the time of autopsy, blood was drawn from the abdominal aorta, and serum levels of 


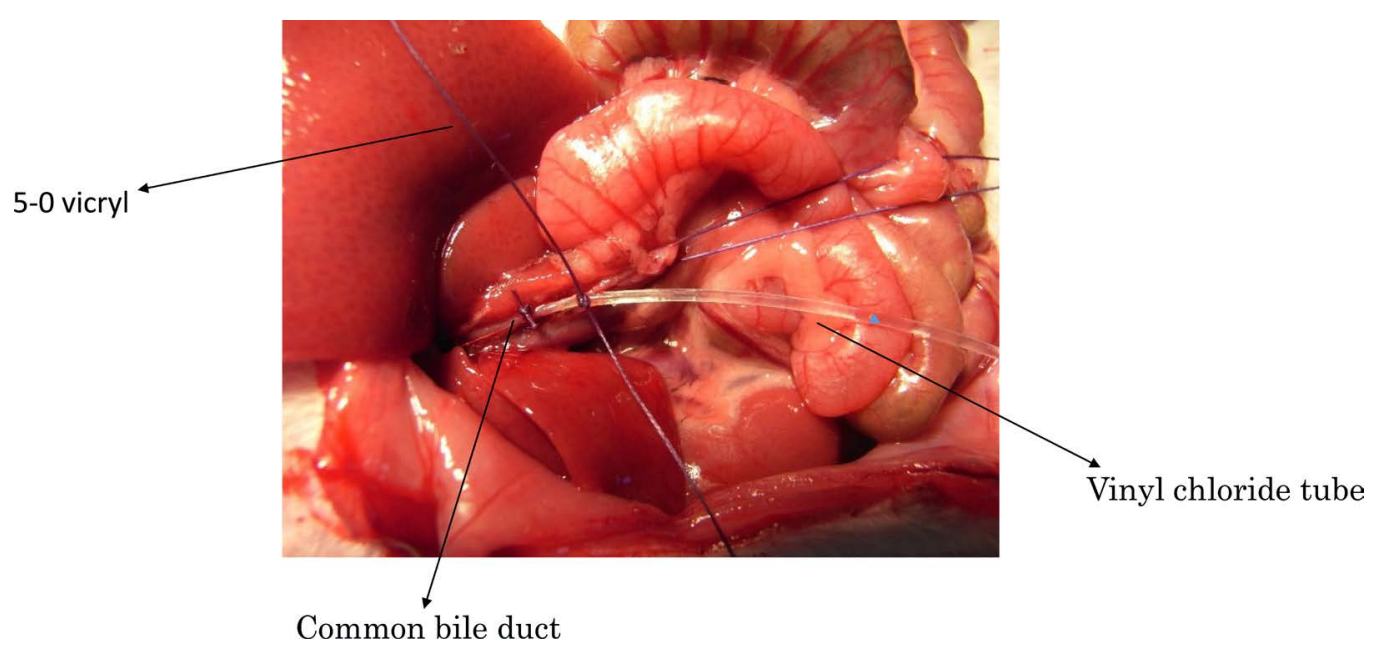

Figure 1. A vinyl chloride stent tube is inserted into the common bile duct, which has been divided proximal to the junction with the pancreatic duct.

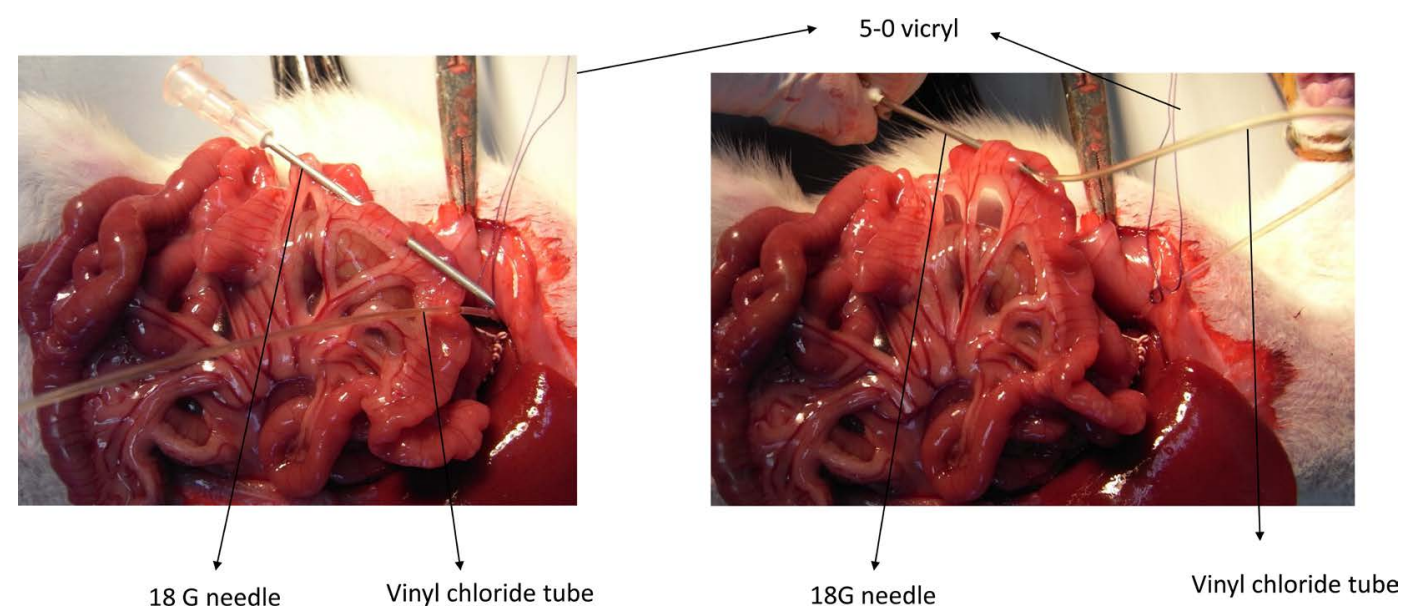

Figure 2. The other end of vinyl chloride tube was inserted into the $18 \mathrm{G}$ needle which was inserted through the jejunum.

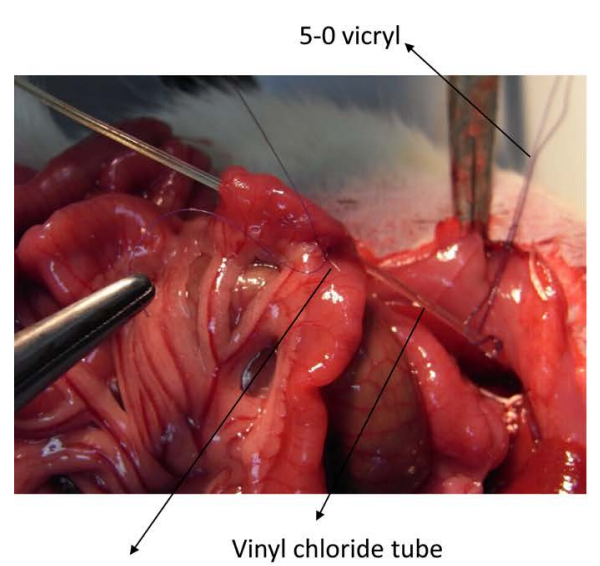

5-0 nylon purse-string suture

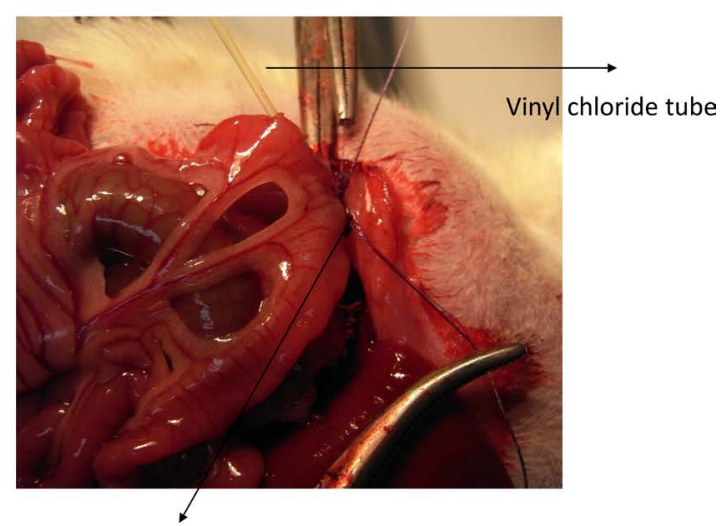

a purse-string suture with 5-0 nylon, which was ligated with 5-0 vicryl in common bile duct.

Figure 3. After the $18 \mathrm{G}$ needle was removed from the jejunum, proximal end of the tube held in place with a purse-string suture with 5-0 nylon, which was ligated with 5-0 vicryl in common bile duct. 


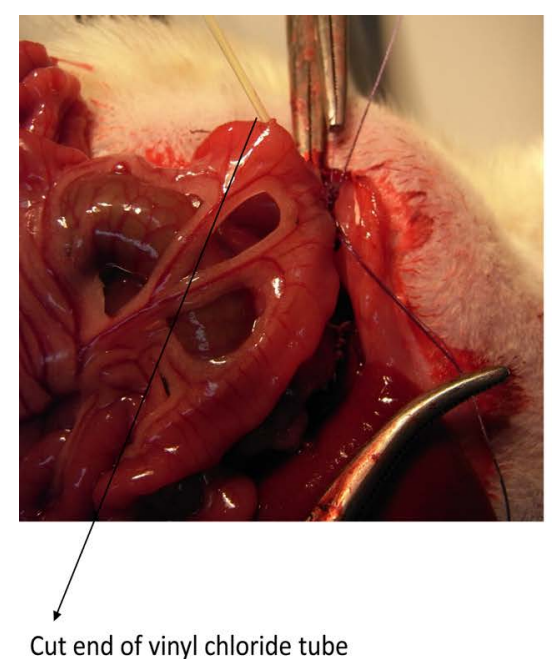

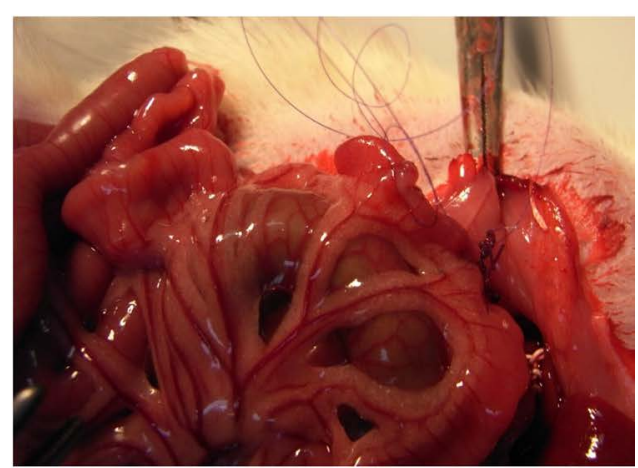

choledochojejunostomy

Figure 4. The cut end spontaneously retracted into the intestinal lumen. And the hole in the jejunum was closed with a stich using 5-0 nylon.

Table 1. Serum biochemical changes in control rat and C-J rat.

\begin{tabular}{ccc}
\hline & Control & C-J \\
\hline Total Bil mg/dl & $0.8 \pm 0.2$ & $1.0 \pm 0.2$ \\
AST IU/L & $96 \pm 5$ & $87 \pm 30$ \\
ALT IU/L & $40 \pm 14$ & $25 \pm 9$ \\
Alp IU/L & $217 \pm 15$ & $871 \pm 100^{*}$
\end{tabular}

${ }^{*} \mathrm{P}<0.05$.

total bilirubin, aspartate aminotransferase (AST), alanine aminotransferase (ALT) and alkaline phosphatase (Alp) were determined using standard autoanalyser methods (Hitachi Autoanalyzer 736; Hitachi Tokyo, Japan).

These procedures were approved by the Animal Care and Facilities Committee, Kindai University.

\subsection{Statistical Analysis}

Data are represented as mean \pm SE. Statistical significance was analysed with Student's paired $t$ and $\mathrm{P}<0.05$ was considered significant.

\section{Result}

\section{1) General course}

Choledochojejunostomy (C-J rat) was successfully performed without technical problems. We could not detect signs of cholangitis or liver abscess in operated rats throughout the entire follow up period.

\section{2) Serum biochemical examination (Table 1)}

There is no difference between control and C-J rat in T-Bil, AST, and ALT. Alp in $\mathrm{C}-\mathrm{J}$ rat was significantly higher than that in control $(\mathrm{P}<0.05)$. 


\section{Discussion}

Bilioenteric anastomosis has played an integral role in the surgical management of biliary tract disease. A wide variety of techniques for suturing a portion of the biliary tract to the digestive tract have been described. Choledochojejunostomy or hepaticojejunostomy is the favoured approach for biliary reconstruction in dilated and thick-walled bile duct. Although accurate mucosa-to-mucosa attachment has been regarded as essential [1], it is very difficult to mucosa-to-mucosa anastomosis in small and fragile bile duct. But several methods for biliary reconstruction without mucosa-to-mucosa alignment have been reported [2]. Cameron et al. [3] reported a procedure of hepaticojejunostomy with a transhepatic tube placed beyond the anastomosis, and they demonstrated that good results could have been obtained in some cases without mucosa-to-mucosa attachment.

Bilioenteric anastomosis in rats is technically difficult, due to the small diameter of the bile duct. Lee [4] had described the method of choledochoduodenostomy in rats for orthotopic liver transplantation. The thread ligating the distal end of the open bile duct was withdrawn along a needle track into the intestinal canal, and was held by fixing the stump of the hepatic artery ligated to the duodenum to minimize tension. We used sutureless anastomosis in a rat experimental model by the modified Lee's technique. The results of the present study indicate clearly that the sutureless anastomosis in a rat model is reliable and leads to long-term anastomotic patency at 12 weeks after surgery.

From the serum biochemical examination, serum bilirubin, AST and ALT in C-J rat was similar to those of control. Alp is an enzyme in the cells lining the biliary ducts of the liver. Therefore, Alp in C-J rat was significantly increased compared to that in control due to stent tube. Therefore there is no problem about the bile flow via choledochojejunostomy in C-J rat.

Our technique is useful when the duct stumps are small and fragile and mucosa-to-mucosa attachment cannot be achieved. When the residual small bile ducts are simply ligated, their drainage areas will become atrophic, with no risk of late biliary stricture or cholangitis. Such a procedure is a reasonable option, provided that hepatic function remains sufficient good. When the patients were considered to have no capacity for sacrificing any liver volume, reconstruction of all residual bile ducts was needed.

Sutureless choledochojejunostomy may have an application in the reconstruction of narrow and fragile bile duct. Our technique will be suitable for the cases in which standard biliary enteric anastomosis is impossible.

\section{Conclusion}

The results of the present study indicate clearly that the sutureless anastomosis in a rat model is reliable and leads to long-term anastomotic patency at 12 weeks after surgery. Our technique will be suitable for the cases of narrow and fragile bile duct which standard biliary enteric anastomosis is impossible.

\section{References}

[1] Rossi, R.L. and Tsao, J.I. (1994) Biliary Reconstruction. Surgical Clinics of North America, 
74, 825-841.

[2] Wexler, M.L. and Smith, R. (1975) Jejunal Mucosal Graft: A Sutureless Technique for Repair of Hilar Bile Duct Strictures. The American Journal of Surgery, 129, 204-211.

https://doi.org/10.1016/0002-9610(75)90299-8

[3] Cameron, J.L., Skinner, D.B. and Zuidema, G.D. (1976) Long-Term Transhepatic Intubation for Hilar Bile Duct Strictures. Annals of Surgery, 183, 488-495.

https://doi.org/10.1097/00000658-197605000-00005

[4] Lee, S., Charters, A.C., Chandler, J.G., et al. (1973) A Technique for Orthotopic Liver Transplantation in the Rat. Transplantation, 16, 664-669.

https://doi.org/10.1097/00007890-197312000-00019

Submit or recommend next manuscript to OALib Journal and we will provide best service for you:

- Publication frequency: Monthly

- 9 subject areas of science, technology and medicine

- Fair and rigorous peer-review system

- Fast publication process

- Article promotion in various social networking sites (LinkedIn, Facebook, Twitter, etc.)

- Maximum dissemination of your research work

Submit Your Paper Online: Click Here to Submit

Or Contact service@oalib.com 